\title{
Monte-Carlo Simulation of Radiation Impact on Flash Memory Cells
}

\author{
E. Dolićanin, D. Vučković, M. Obrenović, I. Fetahović, K. Stanković
}

\begin{abstract}
This paper discusses with the actual problem of radiation effects of ion beams in flash memory cells because of their applicability in control of solar cells in the space, since they are main components of devices which manage all operations of solar cells in the space. The effects of gamma rays may cause changes in stored data, or even the physical destruction of the flash memory components, since the high degree of integrated components miniaturization affects the memory sensitivity. Interaction of ion beam irradiation with the layers enclosing the floating gate, which keep all the information, provides effects that downgrade the memory cell characteristics, primary leads to the changing of the stored content or destruction of the device. Introducing theoretical, experimental and numerical set-up and results using MonteCarlo simulation, has been examined how the cell characteristics in four types of the flash memory affect their sensitivity to ionizing radiation.
\end{abstract}

Keywords: Flash memory, radiation hardness, floating gate, Monte-Carlo simulations.

\section{Introduction}

The high degree of components miniaturization in electronic devices has been introduced into the appearance of the first semiconductor devices and to this day has gained a major level of development in many fields of electrical engineering. The miniaturization of electronic components contains their production by using very thin layers that constitute a composite architecture able to achieve complex functions. Creating electronic devices attending previous approach makes them extremely irresistible to ionizing radiation in the circumstances under which they perform. Thus, remarkably important characteristic of the flash memory components is radiation hardness.

Manuscript received November 29, 2014; accepted April 25, 2015.

E. Dolićanin, M. Obrenović, I. Fetahović are with the State University of Novi Pazar, Novi Pazar, Serbia; K. Stanković is with the Faculty of Electrical Engineering, University of Belgrade, Serbia; D. Vučković, is with the Faculty of Technical Science, University of Priština, Serbia. 
The first experiment was the implementation of the semiconductor devices for the lower frequencies, which did not accomplish acceptable results. A tunnel diode was one of the first semiconductor devices used at lower frequencies, which is now completely restrained by the elements of the TE-diode, bipolar and MOSFET transistors, considering that tunnel diode was proved to be temperature unstable and sensitive to the effects of radiation. In the early sixties, microwave semiconductor devices were developed. Among the abundant usage of semiconductor devices in electronics particular attention should be given to the flash memories.

The latest form of semiconductor memory is flash memory that is named because of the fast access to data and easy reprogramming ability. Flash memory was developed in the mid-eighties in the laboratories of the company Toshiba, and it was in-between EPROM and EEPROM memory. Flash memory uses an electrical erasing technology along with EEPROM. The entire flash memory can be erased in a matter of seconds, but only by blocks [9].

Flash memory bases on a modified MOSFET transistor with insulated gate witch was intermediate between the common gate and substrate offers a fast access to data besides the features that it does not need power supply for data storage. In addition to its characteristics flash memory, packed in a memory card within the device, is almost unbreakable.

Flash memory takes a considerable level of solar cell control in space flights, because they are the main components of devices which manage all operations of solar cells in the space. In this way, the flash memory devices are under the influence of light ions (intense primary cosmic radiation), so the prediction of their applicability is the principal assignment of this research $[8,12]$.

\section{The structure and operation features of the flash memory}

Devices of newer generation are the result of the analysis of the flash memory structure, which can store more than one bit of information per cell (Multi-level cell device), in form of transistor with two input. Each flash memory cell bases its work on modified MOSFET (Metal Oxide Semiconductor Field-Effect Transistor) transistor cell with induced channel which has an additional input, which is isolated with single oxide layer. That inner insulated poly silicon layer is "floating" input (FG), and it interposed between the common input, made of metal layer, usually aluminium, so called control gate $(\mathrm{CG})$ and substrate $(\mathrm{P})$, silicon layer, as shown in Figure 1.

Based on the n-MOSFET transistor with an additional input contact flash memory cell operates with the foregoing amount of electrical charge that is obtained at the gate. Considering that the floating gate is isolated with an oxide layer, each electron appearing in it, is trapped and in that way all successive information is placed on the that floating input. At the boundary voltage in the gate, commonly named the threshold voltage $(\mathrm{Vt})$, the electrons 


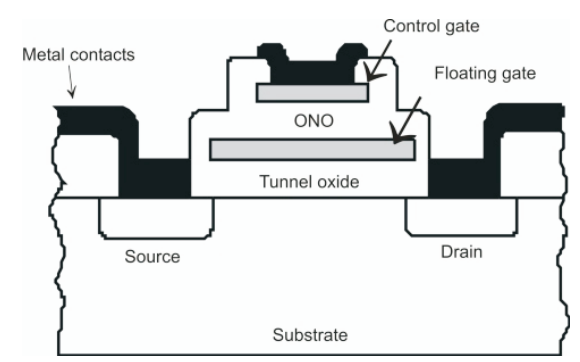

Fig. 1. Flash memory based on n-MOSFET with isolated gate

form a continuous layer, the so-called conductive layer, which is a galvanic connection between the source and drain. Under the influence of a positive drain voltage, the electrons are moving from source to drain conducting a current. Charge stored on the floating gate determines the value of transistor threshold voltage, making the memory cell either "on" or "off" at readout. At a voltage higher than the threshold voltage, concentration of electrons in the conductive layer increases, and the resistance between the source and drain is reduced and under constant drain voltage. Increasing the gate voltage of transistor, current increases, and this correspond to erased transistor with positive or no charge on FG. If increasing the negative gate voltage, channel becomes narrower and the source-drain resistance is gradually increased, wherein the transistor state is programmed with negative charge stored on floating input. Thus, when a cell is "on" or "off" readout by setting certain voltage pulse on the control gate, the current will flow or not flow, relying upon the threshold voltage (Vt) of the cell. The presence or absence of the current electrical condition is observed and translated into " 1 " and " 0 ", in this way reprogramming the stored information, as shown Figure 2 [2].

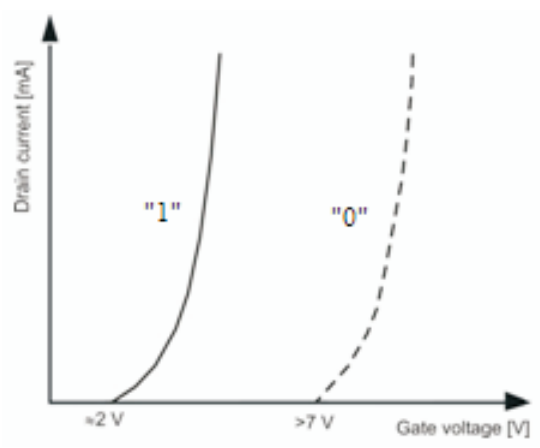

Fig. 2. Flash cells characteristics of the source-drain current as a function of voltage drain-source with induced channel and conducting layer

The function of the insulator is to minimize the leakage of the charge stored at the FG and, at the same time, to provide the best capacitive coupling in the middle the floating 
and the control gate. Medially the floating gate (FG) and the substrate, so-called the tunnel oxide, is commonly amorphous silicon dioxide $(\mathrm{SiO} 2)$ structure. A tunnel insulating layer consists of silicon dioxide, which is one the highest quality materials (Figure3) [8].

The insulator separating the FG and the external gate, CG, is frequently made of an oxide-nitride-oxide (ONO) subsisting of a three-layered structure. The outer layers are made of $\mathrm{SiO} 2$ and a middle layer of silicon nitrite Si3N4, which has good insulating properties, that is shown on Figure 3 [1].

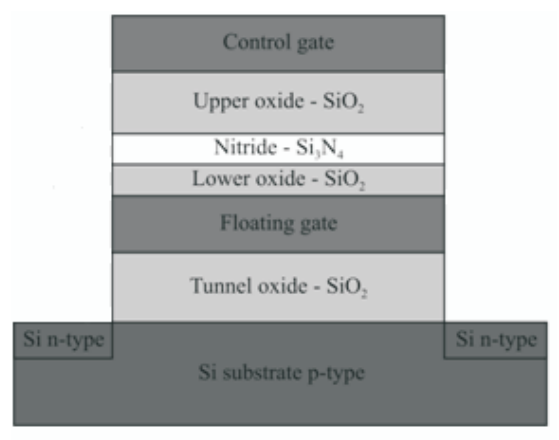

Fig. 3. The structure of the flash memory cell layers used in the simulation.

In terms of its installation in a digital system, there are two categories of the memory. External (portable) NAND is memory with larger capacities. They are supplied as separate components and in a computer are involved via USB (Universal Serial Bus) connectors. Embedded Flash memory components are to be permanently installed in the computer system during production. Embedded memory is usually NOR type, with a relatively small capacity.

Principles of programming and erasing each NOR and NAND memory cells are very different. Programming in a NOR cell technology is performed by injecting "hot" electrons, while the voltage of the external gate leads $(10$ to $12 \mathrm{~V})$, thereby creating an electric field at the surface of the substrate. Drain current leads to a voltage of about $6 \mathrm{~V}$, while source voltage remains at $\mathrm{O} \mathrm{V}$, while the current is large $(0,3-1) \mathrm{mA}$ and flows through the channel between the drain and the source. A bit of the electrons from the current that diffused from $\mathrm{FG}$ have enough energy to overcome the $\mathrm{Si} / \mathrm{SiO} 2$ barrier from substrate to insulator $(3.1 \mathrm{eV})$, go across the insulator, and reach the FG. Voltage used to the CG, constitutes a field cross the tunnel oxide that helps the injection. Hot electron injection is an ineffective method because only about $0.001 \%$ of the current is conducted to the FG. Erasing cells is performed by discharging negative charge of FG, removal of electrons in-source or substrate. In both types of memory, NOR and NAND-type discharge, is performed through the Fowler-Northaim tunneling, which is accomplished by a strong electric field of source or substrate. The control gate is connected to a voltage of $0 \mathrm{~V}(-8 \mathrm{~V})$ while the source voltage 
is $+12 \mathrm{~V}(+5 \mathrm{~V})$ wherein the drain is open.

Memories with NAND architecture are slower, but more efficient and better controlled programming process, based on Cold Electron Tunneling. Voltages for programming and erasing at NAND memories are significantly higher than at NOR memories. It consists of applying a high voltage of a $20 \mathrm{~V}$ to the $\mathrm{CG}$, while the drain, source and bulk are grounded. Tunnel programming requires only a very small channel current less than $1 \mathrm{nA}$, allowing many cells to be programmed at the same time. Erasing NAND cell is performed also by tunnel mechanism, the opposite direction to the one for programming. The control gate is grounded, while the source, drain, and substrate are connected to a high voltage of about $20 \mathrm{~V}$.

The theoretical and experimental radiation resistance of flash memories and their applicability, as well as methods of protection these types of memory, are the main goals of this research.

\section{Monte-Carlo method numerical simulation of the radiation interaction with materials}

Despite the fact the application field of Monte-Carlo methods extended to almost all areas, from radiation and nuclear physics to other fields of science and technology, especially complex geometry, inhomogeneous materials, poly-energy radiation, this technique is a numerical method for solving complex tasks using sequence of random variables on the interval $(0,1)$, remains a considerable and very much applied in radiation physics [10].

Monte-Carlo method implementa-tions in radiation physics are based on the certain problem or correspond to its simulation, therefore the radiation interaction and material are in configuration of stochastic model. The nucleus transformation moment, emission of the particles and directions are not predictable and that makes the particles emission process of ionizing radiation random [15].

The insecurity of the experimental results of the Monte-Carlo method is less than $\pm 5 \%$, and all discrepancies must be considered as uncertainty of calculations or the systematic errors [7].

\section{Experiment}

Flash memory is the leading types of long-term memory, which are used in many applications depending upon electronic parts that work in conditions of radiation. The radiation effects are termed as a effects of Total Ionized Dose (TID). With the accumulation of TID, the flash memory utility decreases $[13,14]$.

The radiation interaction with insulators which envelop the floating input (FG) damages the memory cell performances, increasing the possibility of losing the stored content, and 
damaging or losing the performance functionality.

NOR memories from different manufacturers were subjected to radiation for the purpose of the experiment. Conclusions, which are also valid for NAND flash memories, are made from the analysis of experimental results. The experiment explores the effects of gamma radiation on the cell of four different flash memories. The research of four flash memory models were investigated, all with 512 kbit storage capacity: Numonyx M25P05A (Type 1), Atmel AT25F512B (Type 2), SST SST25VF512 (Type 3), and Macronix MX25L5121E (Type 4). The package TSSOP8 were at memory type 1, although the other three types of memory had 8-lead SOIC packages.

The effect of gamma radiation on the retention of the investigated memory cells and their ability to keep the stored data, when there is no bias, had been examined, so we performed alternately writing " 1 " and " 0 " ("on" and "off") before irradiation, while their other connections had been non-polarized during irradiation.

The radiation was performed in the Laboratory of The Institute of Nuclear Sciences "Vinca", Belgrade, Serbia Irradiation at the irradiation unit of ${ }^{60} \mathrm{Co}$.

Total ionizing dose (TID) ranged from 1 to $12 \mathrm{kGy}$, with an increment of $1 \mathrm{kGy}$, and five samples of each of the four memory types were irradiated at all absorbed dose level in the range. The results presented were obtained as mean values for each of the five samples of the four memory types. The experiments have determined the changes in flash memory of all four types in groups of five samples, compared to absorbed dose which was found to have the highest number of bit error percentage in memory type $1(65 \%)$ at a maximum dose $(12 \mathrm{kGy})$ while the lowest number of bit errors were in memory type $4(42 \%)$. By the end of 9 weeks of irradiation, memory type $4(17 \%)$ had the lowest percentage of remaining errors, while the highest error rate was in memory of type $2(32 \%)$.

\section{Results and discussion}

The vacancy concentration generated the displacing atoms expressed per incident ion have increasing trend regarding to the depth of flash memory cell (Figure 4). This can be defined by the forward relocation of atoms (in the direction of the beam) where at the end of the path of displaced atoms dominate dislocation interactions. At the junction of two materials, which makes difficult vacancy transition from one material to another, exist the energy barrier, due to which increases the vacancy concentration on the distinguish surface layers of cell. The concentration of vacancies for all four types is high in the nitride layer of the ONO dielectric [6]. 


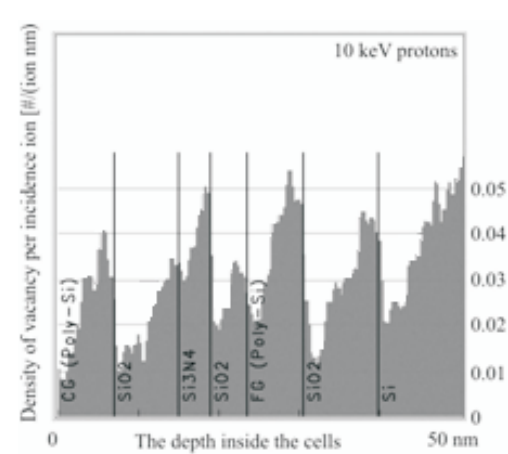

a)

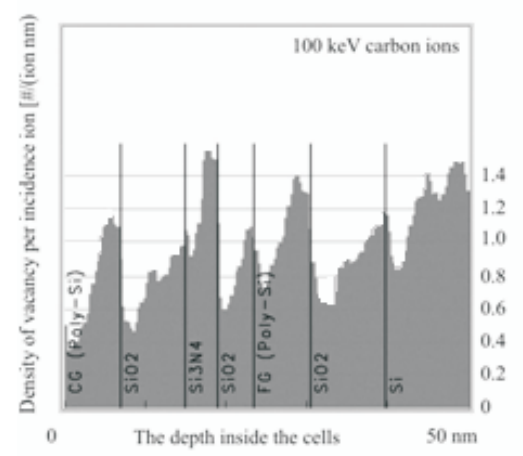

c)

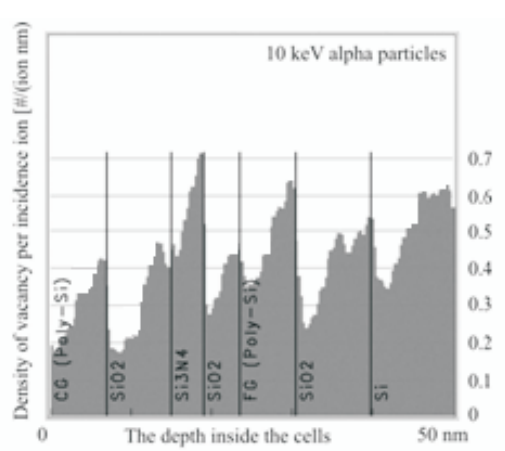

b)

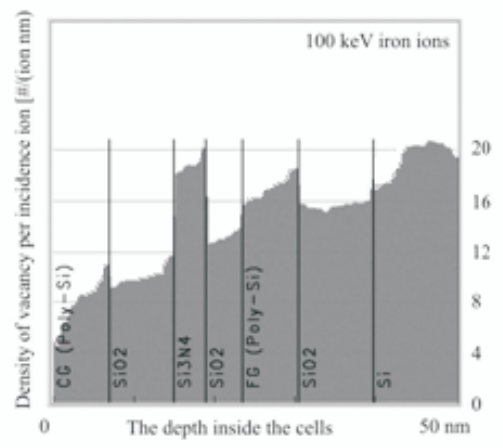

d)

Fig. 4. Vacancy distribution generated by displacing atoms (expressed per incident ion) for: $10 \mathrm{keV}$ protons, b) $10 \mathrm{keV}$ alpha particles, c) $100 \mathrm{keV}$ carbon ions , d) $100 \mathrm{keV}$ iron.

\section{Conclusion}

In the analysis of radiation effect of four commercially applicable flash memory types has been investigated the ability of keeping written data after gamma ray exposure. For purpose of researching the effects as a result of the atom displacements was implemented numerical simulation of the ions passage through the flash memory cell. The bit error percentage rises with the total ionizing dose which is attributed to radiation effects in the tunnel oxide and the isolated layer of the memory cells. Bit error counts did not differ much among various memory models, significance that the sensitivity to gamma ray exposure depends mainly on the dimensions of oxide layers which are approximately equal for the four examined memory models, since they all appeared with the same technology. As a result of the low thickness, the layers of the flash memory cells are insensitive to ion energies greater than approximately $1 \mathrm{MeV}$. The simulation of radiation transport demonstrates that for a given type and energy of ions in some layers of the cells appears great number of displaced atoms and vacancies. Flash cell structure damages caused by such displacement, can affect the 
whole range of its parameters (leakage current, threshold voltage, access time and time of writing data), as well as prevent the re-programming of the cell. Some types of errors may occur during the heavy ion irradiation, even for devices that are not biased. Observing this type of failure mechanism is very useful because of the detection and correction of errors, but also for understand reasons of error appearance within the device, as well as weather internal errors in the memory controller will affect their operation in the space.

\section{Acknowledgement}

The Ministry of Education, Science and Technological Development of the Republic of Serbia supported this work under contracts 171007.

\section{References}

[1] A.Gasperin, A. Paccagnella, G. Ghidini, A. Sebastiani, Heavy Ion Irradiation Effects on Capacitors with $\mathrm{SiO}_{2}$ and $\mathrm{ONO}$ as Dielectrics, IEEE Trans. Nucl. Sci., vol. 56, pp. 2218-2224, 2009.

[2] B. Cavrić, E. Dolićanin, P. Petronijević, M. Pejović, and K. Stanković, Radiation Hardness of Flash Memory Fabricated in Deep-Submicron Technology, International Journal of Photoenergy, Vol 2013, Article ID 158792

[3] G.C. Messenger And M.S. Ash, The Effects of Radiation on Electronic Systems 2nd edition, Van Nostrand Reinhold, New York (1991)

[4] E. DolićAnin et ALl, Gama Ray Effects on Flash Memory Cell Arrays, NTRP,2012, Vol 27, No. 3, pp. 284-289.

[5] J. E. VelazQueZ-Perez, Y. G. Gurevich, Charge-Carrier Transport in Thin Film Solar Cells: New Formulation, International Journal of Photoenergy, Vol. 2011 (2011), Article ID 976063

[6] J.F. Ziegler, J.P. Biersack, M.D. Ziegler, SRIM (The Stopping and Range of Ions in Matter), http://www.srim.org.

[7] K. Stanković, Influence of the Plain-parallel Electrode Surface Dimensions on the type A Measurement Uncertainty of GM Counter, Nuclear Technology \& Radiation Protection (2011), Vol. 26, 39-44

[8] M. Vujisić, K. Stanković, E. Dolićanin, P. Osmokrović, Radiation Hardness of COTS EPROMs and EEPROMs, Radiation Effects and Defects in Solids: Incorporating Plasma Science and Plasma Technology (2010), Vol. 165, No. 5, pp. 362 - 369

[9] M. Vujisić, K. Stanković, A.,VAsić, Comparison of Gamma Ray Effects on EPROM and $E^{2}$ PROMs, Nucl Technol Radiat, 24 (2009),1, pp. 61-67.;

[10] M. Vujisić, K. Stanković, P. Osmokrović, A Statistical Analysis of Measurement Results Obtained from Nonlinear Physical Laws, Applied Mathematical Modeling (2011), Vol. 35 , pp. 3128-3135 
[11] M. Vujisić, N. Marjanović, I. Fetahović, K. Stanković, P. Osmokrović, Influence of Radiation on Titanium Dioxide Mermistors, Scientific Publications of the State University of Novi Pazar

[12] N.S. Marjanović, et al., Simulated Exposure of Titanium Dioxide Memristore to Ion Beams, Nucl Technol Radiat,25(2010), 2, pp. 120-125

[13] M. Zdravković, A. Vasić, R. Radosavluević, M. Vujisić, P. Osmokrović, Influence of Radiation on the Properties of Solar Cells, Nuclear Technology \& Radiation Protection (2011), Vol. 26, No 2, pp. 158-163.

[14] R. Radosavluević, A. Vasić, Effects of Radiation on Solar Cells as Photovoltaic Generators, Nuclear Technology \& Radiation Protection (2012), Vol. 27, No 1, pp. 28-32

[15] Holmes-Siedle AND L. AdAms, Handbook of Radiation Effects, Oxford Science publication, Oxford,(1993). 